\title{
Biología Reproductiva del mangle rojo (Rhizophora mangle L.) Puerto El Morro, Provincia del Guayas, Ecuador
}

\author{
Reproductive biology of the red mangrove \\ (Rhizophora mangle L.) Puerto El Morro, \\ Guayas Province, Ecuador
}

\section{Zoila Peña Villagómez ${ }^{1 *}$ \& Carmita Bonifaz Balseca}

\author{
${ }^{1}$ Consultor independiente, Fundación GAIA, Tlf. 0939809988 \\ 2 Universidad de Guayaquil, Herbario GUAY, Facultad de Ciencias Naturales, Av. Raúl \\ Gómez Lince s.n. y Av. Juan Tanca Marengo (campus Mapasingue), Guayaquil-Ecuador
}

Recibido de agosto 2020; recibido en forma revisada 26 de agosto 2020, aceptado 3 de octubre 2020

Disponible en línea 20 de diciembre 2020

\begin{abstract}
Resumen
El estudio de la Biología reproductiva del mangle rojo (Rhizophora mangle L.) en Puerto El Morro, Provincia del Guayas, Ecuador, tiene por objetivo determinar que la especie es hermafrodita, describiendo sus órganos reproductivos, su estado y proceso de maduración en la flor, desde capullo hasta la antesis, estableciendo las características morfológicas y tamaño de estos. Además, de clasificar el estado reproductivo con respecto a las otras especies de mangle rojo en los manglares de Puerto El Morro, que al momento mantiene pocos árboles de $R$. mangle florecidos y una relación baja de representatividad en los manglares de Puerto El Morro. Para tal efecto, se realizaron dos parcelas correspondientes a 0,25 ha., tanto a la entrada del canal como a la salida del canal de El Morro, lo cual permitió establecer la morfología de $R$. mangle, su densidad y volumen en cada parcela y en relación con las otras especies de mangles rojos presentes.

Palabras claves: Hermafrodita, manglares, morfología, representatividad, Rhizophora mangle.
\end{abstract}

\begin{abstract}
The study of the reproductive biology of the red mangrove (Rhizophora mangle L.) in Puerto El Morro, Guayas Province, Ecuador, aims to determine that the species is hermaphrodite, describing its reproductive organs, its state and maturation process from flower bud to anthesis, establishing the respective morphological characteristics and sizes. In addition, to classify the reproductive status with respect to the other species of red mangrove in Puerto El Morro mangroves, which currently maintains few flowered $R$. mangle trees and a low representativeness ratio in the mangroves of Puerto El Morro. For this purpose, two plots corresponding to 0.25 ha were made, both at the entrance and exit of the canal El Morro channel, which allowed establishing the morphology of $R$. mangle, its density and volume in each plot, and in relation to the other related species of red mangroves present.
\end{abstract}

Keywords: hermaphrodite, mangroves, morphology, representativeness, Rhizophora mangle.

\section{Introducción}

Se considera a los manglares entre los ecosistemas de mayor importancia de la biósfera, con significativos aportes de bienes y servicios a nivel mundial. Se encuentran en zonas cálidas, desarrollándose principalmente donde hay esteros, bahías y deltas de ríos que desembocan al mar (Charcape-Ravelo \& Moutarde, 2005).

En América se distribuyen del lado del Océano Pacífico desde los $30^{\circ} 15^{\prime} \mathrm{N}$ hasta los $5^{\circ} 34^{\prime} \mathrm{S}$, esto incluye desde el noroeste de México en el estado de Sonora hasta el departamento de Piura en Perú; del lado del Atlántico se distribuye desde la zona norte de Florida en Estados Unidos hasta el sur de Brasil desde aproximadamente $30^{\circ} \mathrm{N}$ hasta los $25^{\circ} \mathrm{S}$ (Charcape-Ravelo \& Moutarde, 2005).

Los bosques salados o humedales costeros forman un tipo de asociación vegetal halófila, constituida por árboles perennifolios con raíces fúlcreas, las mismas que le dan una fisionomía arquitectónica muy típica. Colonizan zonas que se encuentran anegadas y 
según el tipo de ubicación en las costas tropicales y subtropicales; se los denominan manglares ribereños, de borde y de cuenca (Ortiz-Reyes et al., 2018).

Son ecosistemas de importancia por su gran producción de biomasa y energía (carbono orgánico). La materia orgánica que cae es degradada con mucha facilidad, sirviendo de nutrientes para los organismos que lo habitan. Además, los manglares contribuyen a la mitigación del cambio climático, debido a que constituyen sumideros de carbono (Lozano Torres, 2007).

Estas asociaciones arbóreas se desarrollan en suelos fangosos, anaeróbicos, en aguas de alta salinidad - salobres, mareas cambiantes, ingresando en ocasiones hasta el interior continental donde el medio sea apropiado para su óptimo desarrollo. Están constituidos por especies vegetales particulares que cuentan con adaptaciones morfológicas y fisiológicas para su desarrollo en condiciones extremas, siendo plantas altamente resistentes a la salinidad (De la Cruz-Francisco, 2012).

Entre los servicios ambientales que proveen sirven de barreras para los vientos, control de inundaciones, evitan la erosión, resisten baja concentraciones de oxígeno y suelos inestables, y son de gran importancia económica, cultural y científica. Estos ecosistemas costeros poseen una gran productividad, son la base principal del ciclo de vida de una enorme diversidad biológica de flora y fauna; brindan hábitat, refugio, son sitios únicos de anidación y alimentación para una gran diversidad de especies marinas y terrestres, necesarios para el desove y crianza de vertebrados como peces, anfibios, reptiles, aves y mamíferos, e invertebrados como crustáceos, moluscos, etc... (Ortiz-Reyes et al., 2018).

Conocer el funcionamiento natural del ecosistema manglar con sus procesos reproductivos, además de sus servicios ambientales de regulación ecosistémica, ha contribuido al conocimiento de estos para la sostenibilidad de sus poblaciones (Yánez-Arancibia et al., 1999; Farnsworth et al., 1996).

Los mangles se adaptan a un tipo generalizado de polinizadores, la capacidad de los mangles para convertir las visitas florales en la fertilización de flores y la producción de frutos es un proceso muy importante y necesario para el mantenimiento de estos biomas existentes (Raju, 2016). La biología reproductiva de los mangles presenta características pioneras como la autocompatibilidad y la utilización de una variedad de polinizadores, distribución amplia y semillas de amplio radio de dispersión (Tarcila \& Machado, 2014).

Los mangles comprenden aproximadamente 70 especies agrupadas en 16 familias alrededor del mundo (Ortiz-Reyes et al., 2018). Rhizophoraceae es una de las familias que caracterizan a los manglares, sus flores son principalmente anemófilas, aunque también son visitadas con poca frecuencia por insectos como abejas, mariposas, y hasta aves. Las flores de Rhizophoraceae poseen pistilo y androceo, es decir son hermafroditas, siendo su polen disperso principalmente por el viento (CABI, 2019).

En Ecuador, los manglares ocupan aproximadamente 157.094,28 ha, florísticamente se encuentran caracterizados por especies de mangles mayores: Rhizophora mangle, $R$. racemosa, $R$. x harrisonii, Laguncularia racemosa var. racemosa, L. racemosa var. glabriflora y Hilairanthus germinans (Cornejo, 2014).

Estudios florísticos, estructurales y taxonómicos han sido realizados para Rhizophoraceae a lo largo del Neotrópico, Pacífico y Centroamérica (Prance, 2018; Jiménez, 2004); además de estudios de polinización influenciados por periodos lluviosos y viento en relación con su incidencia en la producción de flores (Sánchez Núñez, 2015; Tarcila y Machado, 2014).

En Ecuador se han llevado a cabo algunas observaciones sobre el desarrollo del manglar que han contribuido al conocimiento florístico y estudios taxonómicos han permitido conocer sobre las especies que conforman las asociaciones vegetales de manglar (Cintrón et. al 1981; Cornejo, 2014). Sin embargo, no hay muchas investigaciones sobre la biología reproductiva de las especies que caracterizan al manglar, aunque algunos trabajos mencionan observaciones de la biología reproductiva en particular de Rhizophora mangle L., indicando aspectos de su fenología y descripciones morfológicas (Cornejo 2014, Ministerio del Ambiente 2010). Sin embargo, estudios de la biología reproductiva de las especies de mangle son requeridos.

El comportamiento reproductivo en $R$. mangle, ha sido descrito desde su capullo floral hasta el momento en que comienza la antesis, pasando por un proceso progresivo de días para la apertura de la flor y maduración de sus órganos reproductivos, la misma, aunque es hermafrodita y protándrica, no se encuentra receptiva al mismo tiempo (Tarcilia Nadia et al., 2014; Cornejo, 2014).

La capacidad reproductiva entendida como la magnitud de floración relacionada con el tipo fisiográfico de manglar y la especie de mangle, ha permitido entender cuántas flores se transformarán en frutos y propágulos (Sánchez Núñez, 2009).

El presente estudio se enfoca particularmente en analizar la biología reproductiva de $R$. mangle en los manglares de Puerto El Morro, en la provincia del Guayas, en la costa de Ecuador. Los resultados permitirán esclarecer la identidad reproductiva de esta especie en los manglares de borde de este sector, además de clasificar su biología reproductiva dentro de cuadrantes permanentes a la entrada y salida del Canal del Morro, permitiendo establecer si $R$. mangle es la especie dominante en la fisonomía de estos 
Rev. Cient. Cien. Nat. Ambien. 14(2):182-189 Diciembre 2020
Peña \& Bonifaz • Biología Reproductiva del mangle rojo (Rhizophora mangle L.) Puerto El Morro, Provincia del Guayas, Ecuador manglares. Los resultados que se presentan son una contribución a la conservación de sus poblaciones en las áreas naturales de manglar del país y un aporte a la conservación y manejo de los mangles rojos.

\section{Materiales y Métodos}

\section{Área de estudio}

El presente trabajo se realizó en Puerto El Morro localizado a $80^{\circ} 19^{\prime} 33.24^{\prime \prime} \mathrm{W}$ y $2^{\circ} 38^{\prime} 5.1^{\prime \prime} \mathrm{S}$, con un rango altitudinal de 0 - 28 metros, ubicado en el Recinto Puerto el Morro a 2 horas de la ciudad de Guayaquil en la provincia del Guayas, a $9 \mathrm{Km}$. del balneario General Villamil-Playas, en la parte norte del Golfo de Guayaquil muy cerca de la isla Puná donde inicia el canal El Morro, de 3 kilómetros de ancho, que es la vía de acceso de las embarcaciones al golfo y al puerto de Guayaquil

Posee 10.130,16 hectáreas de extensión que incluyen bosques de mangles, esteros y canales naturales. El clima de Puerto El Morro es desértico tropical, durante el año hay poca lluvia, la temperatura promedio anual se encuentra entre $23^{\circ}$ y $25^{\circ} \mathrm{C}$., con una precipitación aproximada de $500 \mathrm{~mm}$ al año (Soto, 2010). Tiene dos sectores: la parte continental que abarca 2.094 hectáreas alrededor de los esteros El Morro y el Salado, y una parte insular la que abarca aproximadamente 8.000 hectáreas, comprendiendo un complejo de islas, y formaciones rocosas llamados Farallones del canal de Morro (MAE, 2014). En estos manglares se establecieron dos parcelas, la una a la entrada del canal y la otra a la salida del canal del Morro (Fig. 1).

\section{Metodología}

\section{Metodología de Campo}

Para analizar la biología reproductiva de Rhizophora mangle en Puerto El Morro se procedió a establecer dos parcelas de $50 \times 50 \mathrm{~m}^{2}$ en un sector hacia adentro del Canal El Morro llamado Estero Olmos y la otra parcela hacia afuera del Canal El Morro denominado la Islita, con la finalidad de levantar la información requerida, en áreas muestréales de 0,25 hectáreas de manglar, pero en distintos sectores del ecosistema manglar de Puerto El Morro.

En cada parcela se procedió a georreferenciar cada punto de la parcela con un GPS Garmin la cual fue delimitada (encerrada) completamente por una cuerda de nylon (Fig. 2), una vez realizado esto se comenzó la marcación de cada individuo del cuadrante con fichas plásticas, las mismas que se las amarró en cada árbol.

Las medidas dasométricas, tomadas constituyen el diámetro (Diámetro a la altura del pecho o DAP) y la altura total de cada árbol de mangle, el DAP fue medido con una cinta diamétrica, la misma que tiene marcada la medida directa de superficie, la altura fue estimada en función de referencias objetivas.

Una vez que fueron numerados todos los árboles de la parcela y debido al complejo sistema formado por la arquitectura de las raíces de los mangles, se utilizó binoculares para observar la inflorescencia de $R$. mangle, debido a la dificultad del desplazamiento

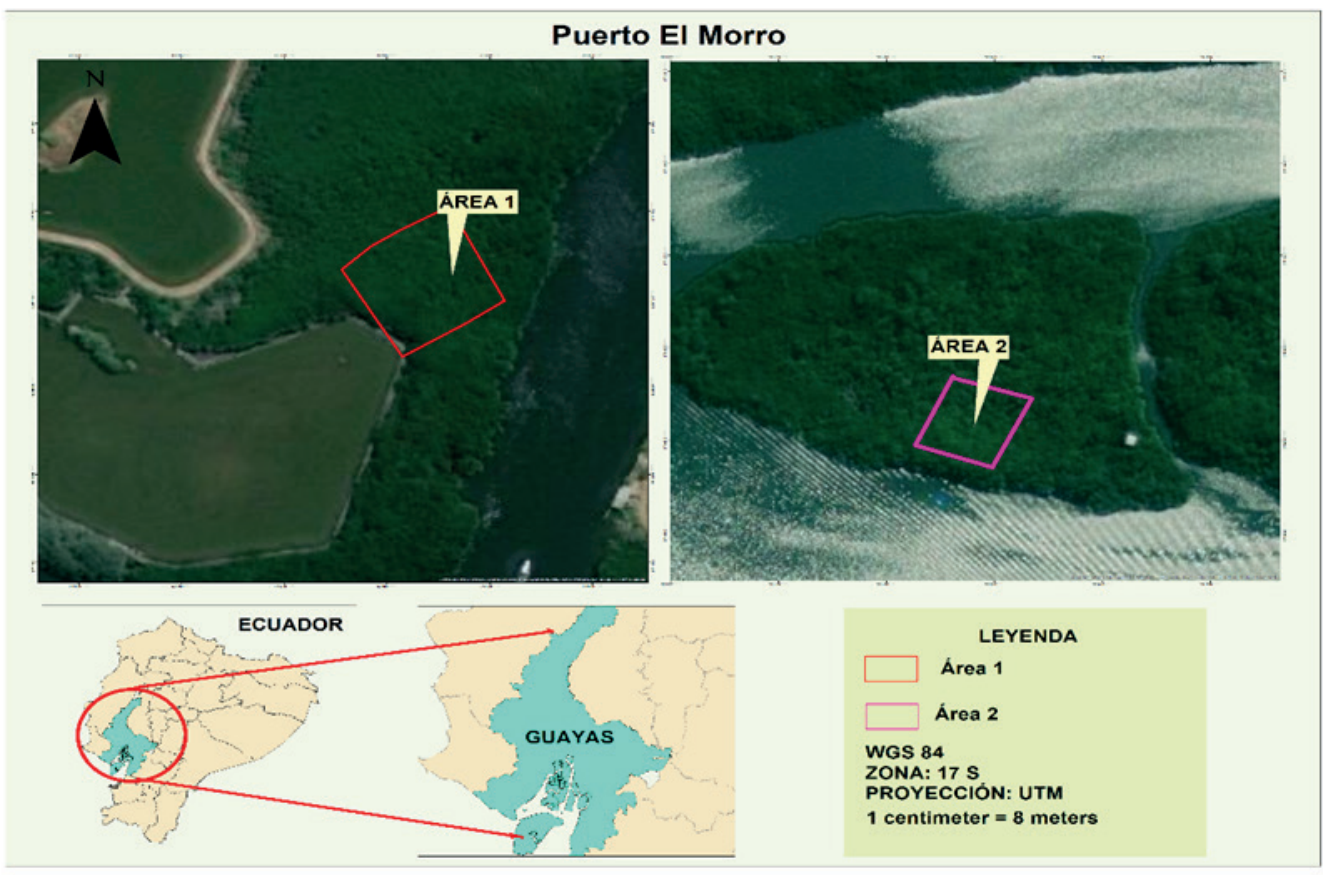

Figura 1. Ubicación de las dos parcelas en Puerto El Morro. Fuente: Eva Moncada 2020. 


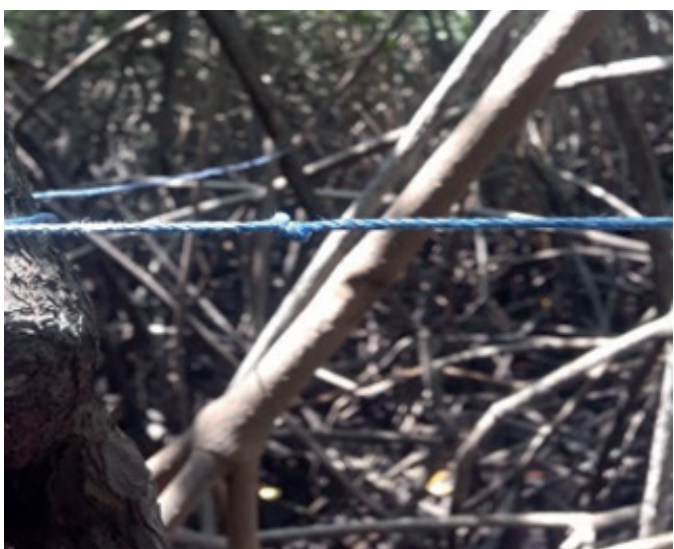

Figura 2. Delimitación de Parcela

entre las raíces, para luego proceder a recolectar las muestras de flor de los árboles respectivos.

En campo, se utilizó estrategias de observación, debido a que en el mismo cuadrante había otras especies de mangle y fisonómicamente todos los árboles se parecen. La diferencia se enfocó entre especies de mangle, por características morfológicas muy particulares como su inflorescencia a nivel de pedicelos, ya que $R$. mangle posee pedicelos articulados (2) con pocas flores, mientras que $R$. racemosa presenta pedicelos articulados (3) con mayor cantidad de flores y el híbrido $R$. $x$ harrisonii presenta pedicelos (4) con abundantes flores. Se elaboró una clave para la observación de campo de las Rhizophora destacando su número de flores y tamaño. Adicionalmente de cada parcela, se recolectó muestras de agua y lodo en ambos sectores en pleamar y bajamar, para tener los valores de salinidad de agua y salinidad intersticial del lodo.

\section{Metodología de Laboratorio}

Las muestreas de flores de $R$. mangle, previamente recolectadas y preservadas en frascos con alcohol de $70^{\circ}$, se las llevó al laboratorio y con ayuda de un estereoscopio se procedieron hacer los cortes histológicos y las mediciones respectivas de los diferentes estadios reproductivos (masculino y femenino) en cada una de las flores y en cada inflorescencia, procediendo a realizar cortes exactos transversales y longitudinales para cada una de las partes reproductivas.

Los cortes transversales y longitudinales se realizaron en flores tanto a nivel de prefloración o capullo floral, así como en flores abiertas. Se observó el perianto, tomando medidas de los tamaños de sépalos y pétalos. A nivel de gineceo se observó la posición del ovario con respecto al cáliz, se realizó la medición de los óvulos, cuantificación de su número y verificación de su estado de madurez; en los carpelos se observó la cantidad de óvulos y la ubicación de estos en el carpelo. Los cortes longitudinales permitieron observar los óvulos y su placentación. A nivel de androceo, se pudo observar el estado de los estambres y anteras

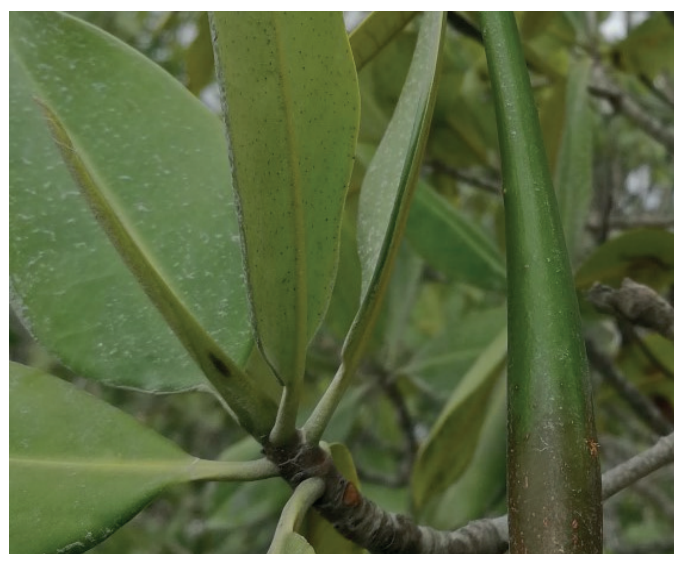

Figura 3. Estipula terminal de Rhizophora mangle.

presentes. Las mediciones también fueron hechas en su estructura externa a nivel de la inflorescencia (flores y prefloraciones) pedúnculos, pedicelos, hojas, y estípulas terminales (Fig. 3).

\section{Resultados}

Descripción de características reproductivas del Rhizophora mangle

\section{Resultados de campo}

Las característica reproductivas observables fueron descritas con una clave sencilla elaborada en base a las caracteristicas morfológicas observadas en campo, la misma que permitió diferenciar en campo las especies de Rhizophora dentro de la parcela, esta se presenta a continuación.

Clave elaborada para reconocer Rhizophora mangle en campo

1. Flores 2 a 4 por inflorescencia, cada una de aproximadamente $1 \mathrm{~cm}$............................... mangle

2. Flores 5 o más por inflorescencia

2.1 Flores de $1 \mathrm{~cm}$ (medianas).............R. racemosa

2.2 Flores hasta $1.5 \mathrm{~cm}$ (grandes)...R. x harrisonii Elaborada: Peña, 2020

\section{Descripción morfológica de campo de Rhizophora mangle}

Hojas opuestas, simples, glabras; peciolos de 1.2 a $3 \mathrm{~cm}$ de largo; lámina elíptica hasta oblonga, 8 a 10 $\mathrm{cm}$ de largo y 4 a $5 \mathrm{~cm}$ de ancho, ápice obtuso, color verde-olivo, lustrosas en el haz, agrupadas hacia la parte terminal de la rama; estípulas 2, terminales, envueltas entre sí en disposición estrechamente cónica, lustrosas, lanceoladas, de 4 a $6 \mathrm{~cm}$ de largo, con presencia de coléteres basales en su interior, dejando cicatrices anulares en las ramas al caer. Flores protándrica, lo cual se puede observar a simple vista, el androceo siempre se encuentra maduro mientras que el pistilo no presenta receptividad. 
Rev. Cient. Cien. Nat. Ambien. 14(2):182-189 Diciembre 2020
Peña \& Bonifaz • Biología Reproductiva del mangle rojo (Rhizophora mangle L.) Puerto El Morro, Provincia del Guayas, Ecuador

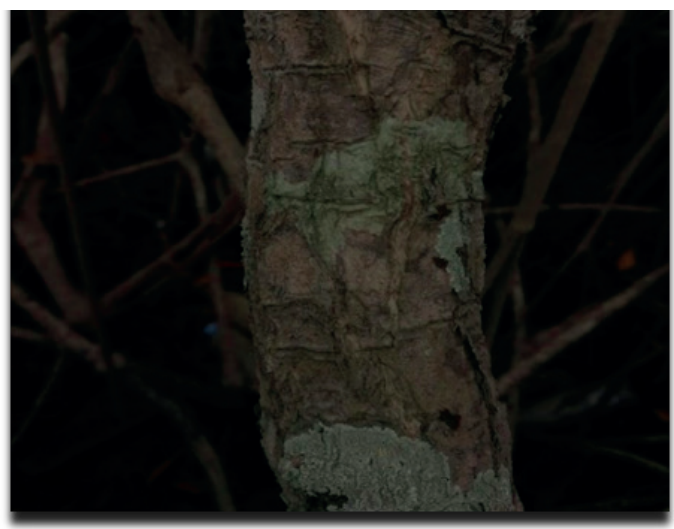

Figura 4. Corteza de R. mangle en Puerto El Morro.

En el área de estudio objetivamente Rhizophora mangle es una especie que presenta árboles de tamaño mediano, con raíces aéreas en forma de zancos arqueadas o fúlcreas y adventicias. Los troncos son rectos con corteza rugosa gris o marrón y con presencia de placas que se observan en forma de escamas, siendo su parte interna de color rojo (Fig. 4).

\section{Resultados de Laboratorio}

Las Inflorescencias colectadas en los individuos de $R$. mangle presentes en las parcelas son cimosas y en un proceso progresivo de apertura de la flor (Fig. 5), poseen de 2 a 4 flores, de 4 a $7 \mathrm{~cm}$ de largo, pedúnculo de 2 a $5 \mathrm{~cm}$, pedicelo de 1 a $2 \mathrm{~cm}$ de largo (Anexo 1). Los capullos florales poseen forma ovadaelíptica con ápice agudo, presentan 5 a $8 \mathrm{~mm}$ de largo; son hermafroditas (Anexo 2), poseen Hipantio (Fig. 6), de $2.5 \mathrm{~mm}$. El cáliz está constituido por 4 sépalos, cada uno de $4 \mathrm{~mm}$ de ancho por $9 \mathrm{~mm}$ de largo, coriáceos, lanceolados, glabros. La corola posee 4 pétalos libres de $4 \mathrm{~mm}$ de largo, lanceolados, abundante pubescencia marginal. El androceo posee 8 estambres, 4 estambres son epipétalos y 4 estambres son alternipétalos, las anteras son sésiles, de 3.5 a 5 $\mathrm{mm}$ de largo. El gineceo posee estigma bifurcado de 5 $\mathrm{mm}$, estilo cónico, ovario semi-inferior de $3 \mathrm{~mm}$, los óvulos son 4, de color crema; los carpelos son 2 de 2 $\mathrm{mm}$, fusionados, la placentación es apical (Anexo 3).

Cuando el estigma bifurcado está receptivo los óvulos presentan una forma redondeada, grande y de color

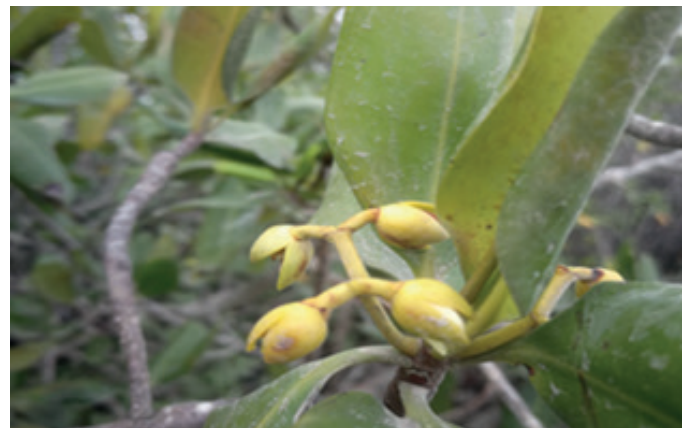

Figura 5. Inicio de la antesis en R. mangle. crema y cuando el estigma no está receptivo, el ovario y los óvulos son de tamaño inferior en comparación cuando están maduros.

La salinidad fue medida con un salinómetro o refractómetro, en las muestras de agua recolectadas en las dos parcelas, tanto en pleamar como en la bajamar y del lodo para su análisis intersticial, dando en los dos cuadrantes una salinidad de 32 ppm en bajamar y 30 ppm en pleamar. La salinidad intersticial del suelo es de 35 ppm.

\section{Clasificación de la Biología reproductiva del Rhizophora mangle}

Las dos parcelas permanentes establecidas en los manglares del Puerto el Morro representan unidades muestréales de 0,25 hectáreas, la una en el Estero Olmos en la entrada del canal, y la segunda en el sector la Islita en la salida del canal, con un total de 34 (Anexo 4) y 18 (Anexo 5) individuos respectivamente. En la parcela del Estero Olmos el 70\% de los mangles presentan categorías diamétricas entre 7 a $13 \mathrm{~cm}$ de DAP (Tabla 1), poseen una fisonomía de árboles delgados con muchas raíces fúlcreas entrelazadas. En cuanto a la distribución los individuos están repartidos desde el borde del manglar en transición a tierra más firme hacia adentro del área de suelo con una típica formación de manglar de borde; con individuos que forman un área basal de $1.8 \mathrm{~m}^{2}$, denotando una densidad elevada de individuos de mangles jóvenes (Fig. 7).

El 47\% de los individuos presentes en la parcela poseen alturas entre 9 a 11 metros, con un volumen total de $21 \mathrm{~m}^{3}$ (Tabla 2).

Correspondiendo a $R$. mangle el $9 \%$ (Gráfico 1) de los individuos presentes en la parcela, con categorías diamétricas que van de 6.5 a $8.2 \mathrm{~cm}$ de DAP, y con alturas que van de 8 a 10.5 metros, con un área basal de $0.085 \mathrm{~m}^{2}$ y con un volumen total de $0.832 \mathrm{~m}^{3}$, siendo su representatividad de $4,5 \%$ en la parcela.

En la parcela de la Islita (afuera del canal del Morro) el $39 \%$ de los mangles presentan categorías diamétricas entre 20 a $24 \mathrm{~cm}$ de DAP, con un área basal de 1.7 $\mathrm{m}^{2}$ (Tabla 3). Los individuos presentan un patrón de distribución continuo desde un borde hasta otro

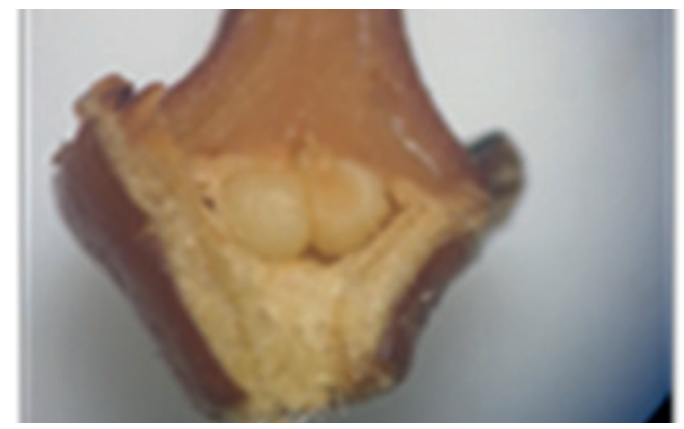

Figura 6. Hipantio de R. mangle. 
Rev. Cient. Cien. Nat. Ambien. 14(2):182-189 Diciembre 2020
Peña \& Bonifaz • Biología Reproductiva del mangle rojo (Rhizophora mangle L.) Puerto El Morro, Provincia del Guayas, Ecuador
Tabla 1. Área basal de los individuos, Estero Olmos.

\begin{tabular}{ccc}
\hline $\begin{array}{c}\text { Clase } \\
\text { diamétrica }\end{array}$ & $\begin{array}{c}\text { Número de } \\
\text { árboles }\end{array}$ & $\begin{array}{c}\text { Área basal total de la clase } \\
\text { diamétrica }\left(\mathrm{m}^{2}\right)\end{array}$ \\
\hline $0-6.99$ & 1 & 0,027 \\
$7-13.99$ & 24 & 1.053 \\
$14-20.99$ & 6 & 0.403 \\
$21-27.99$ & 2 & 0.213 \\
$28-34.99$ & 0 & 0 \\
$35-41.99$ & 1 & 0.203 \\
& 34 & 1.872 \\
\hline
\end{tabular}

Tabla 2. Volumen total de los individuos, Estero Olmos

\begin{tabular}{rrr}
\hline \multicolumn{1}{c}{ Altura } & $\begin{array}{c}\text { Número } \\
\text { de árboles }\end{array}$ & Volumen $\left(\mathrm{m}^{3}\right)$ \\
\hline $0-2.99$ & 0 & \\
$3-5.99$ & 0 & \\
$6-8.99$ & 7 & 1.765 \\
$9-11.99$ & 16 & 7.408 \\
$12-14.99$ & 11 & 11.958 \\
\hline & 34 & 21.131 \\
\hline
\end{tabular}

Tabla 3. Área basal de los individuos, la Islita.

\begin{tabular}{ccc}
\hline $\begin{array}{c}\text { Clase } \\
\text { diamétrica }\end{array}$ & $\begin{array}{c}\text { Número } \\
\text { de } \\
\text { árboles }\end{array}$ & $\begin{array}{c}\text { Área basal total de la } \\
\text { clase diamétrica }\left(\mathrm{m}^{2}\right)\end{array}$ \\
\hline $0-4.99$ & 0 & \\
$5-9.99$ & 1 & 0.021 \\
$10-14.99$ & 3 & 0.156 \\
$15-19.99$ & 6 & 0.511 \\
$20-24.99$ & 7 & 0.900 \\
$25-29.99$ & 1 & 0.123 \\
& 18 & 1.711 \\
\hline
\end{tabular}

Tabla 4. Volumen total de los individuos, La Islita.

\begin{tabular}{|c|c|c|}
\hline Altura & $\begin{array}{l}\text { Número } \\
\text { de } \\
\text { árboles }\end{array}$ & Volumen $\left(\mathrm{m}^{3}\right)$ \\
\hline $0-3.99$ & 0 & \\
\hline $4-7.99$ & 2 & 0.378 \\
\hline $8-11.99$ & 5 & 3.774 \\
\hline $12-15.99$ & 6 & 6.776 \\
\hline \multirow[t]{2}{*}{$16-19.99$} & 5 & 12.423 \\
\hline & 18 & 23.351 \\
\hline
\end{tabular}

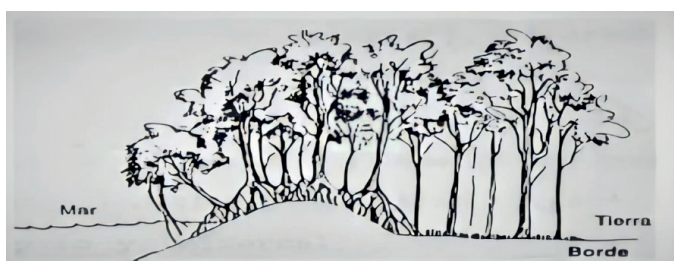

Figura 7. Perfil fisionómico de manglar de borde de Puerto. El Morro, Estero Olmos.

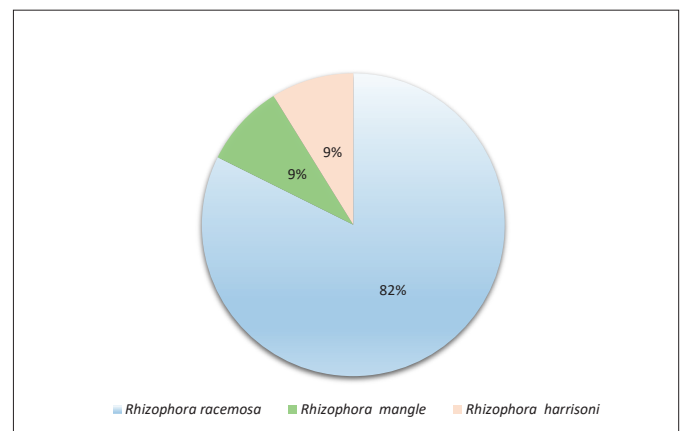

Figura 8. Especies presentes, parcela 1, Estero Olmos.

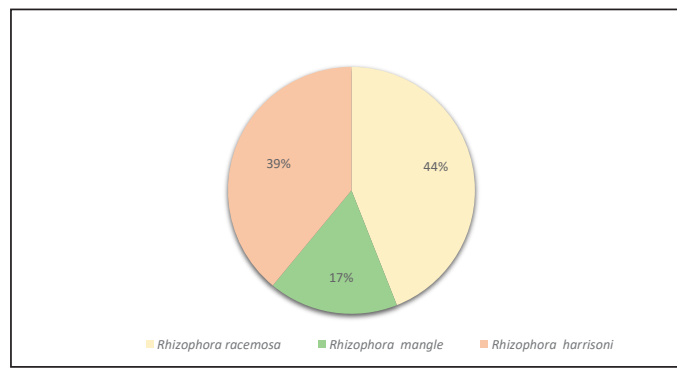

Figura 9. Total, de especies presentes, parcela 2, La Islita.

borde, no hay transición hacia tierra firme, dándole una fisonomía de árboles un poco más gruesos que los de El Estero Olmos, siendo muy parecido el aspecto de entrelazamiento de sus raíces, aunque con un desarrollo un poco mayor (Fig. 8).

El 33\% de individuos de mangle presenta alturas entre 2 a 15 metros, siendo su área basal total o densidad de $1.7 \mathrm{~m}^{2}$ y su volumen total de $23 \mathrm{~m}^{3}$ (Tabla 4).

Correspondiendo a $R$. mangle el $17 \%$ (Gráfico 2) de los individuos presentes en la parcela con categorías diamétricas que van de 22 a $28 \mathrm{~cm}$ de DAP, y con alturas que van de 9 a 12 metros, con un área basal de $0.339 \mathrm{~m}^{2}$ y su volumen total de $3.342 \mathrm{~m}^{3}$, siendo su representatividad de $20 \%$ en la parcela.

\section{Discusión}

En Rhizophora mangle la cantidad de flores en su inflorescencia y su morfología hermafrodita ha sido un carácter utilizado para describir la especie (Prance, 2018; Cornejo, 2014). Siendo, inadecuado reportar la existencia de árboles de mangle hembra 
y mangle macho (Hilgert y Cárdenas, 2004, en el Plan de Manejo de Manglares El Morro), presumiendo que dicha especie es monoica. Por lo cual con el presente trabajo queda demostrado que las flores de R. mangle son hermafroditas.

Hablar de la biología reproductiva en Rhizophora mangle involucra el desarrollo del hipantio en flores con ovario semi-infero desarrollándose a supero (Cornejo 2014), dicha característica es observable en los cortes longitudinales realizados en las flores analizadas de esta especie. El ovario semi-infero es más obvio observar cuando se retiran los sépalos que cubren a la flor y, además, se puede apreciar el anillo que circunda al ovario.

Cornejo, 2014 describe que en $R$. mangle la maduración de los estambres se produce mientras se encuentra en el capullo floral, indicando que la coloración externa de la prefloración tiene una progresión de color verde a color banana. Sin embargo, en los manglares de Puerto El Morro, las prefloraciones se mantienen verdes y sus estambres en condición de maduración abierta una vez abierta la flor.

Algunos han indicado que la magnitud de floración se encuentra relacionada con el tipo fisiográfico de manglar y la especie de mangle (Sánchez Núñez, 2009), lo cual debería ser mejor analizado, pues en los manglares de Puerto El Morro es más dependiente de la especie de mangle y el periodo.

Aunque, el presente estudio se realizó en un lugar del Golfo de Guayaquil, como los manglares de Puerto El Morro, sin embargo, la dinámica de floración podría estar supeditada a los periodos de más o menos lluvia que a la fisiografía del manglar, jugando un papel muy importante el desarrollo del manglar en la incidencia de su capacidad reproductiva.

\section{Conclusiones}

Rhizophora mangle es una especie hermafrodita, sus flores presentan ambos sexos gineceo y androceo en la misma flor.

Rhizophora mangle posee flor protándrica, pues el androceo madura antes que el pistilo.

En Rhizophora. mangle el hipantio está presente en la estructura de las flores de esta especie tanto con ovario semi-infero como con ovario infero.

Rhizophora mangle presenta cuatro óvulos de color crema, dos en cada lóculo.

La prefloración se mantiene de un color verdoso y no presentó variación de coloración hacia amarillo para indicar la madurez del capullo floral.

Los individuos de Rhizophora mangle presentan poca floración en el periodo seco del mes de agosto.

Rhizophora mangle no caracteriza fisonómicamente a los manglares de Puerto El Morro, esto se concluye por la cantidad de individuos presentes en las parcelas muestreadas y su área basal individual y total en los dos sectores del área de estudio, en relación con el número total de individuos.

En cuanto a la fisonomía, los manglares de Puerto El Morro muestran mangles jóvenes hacia la parte interior del canal y con muchas raíces entrelazadas dando un aspecto muy denso (Anexo 6), y mangles más desarrollados hacia afuera del canal del Morro, por lo cual es razonable entender que dicha fisonomía está dada por la función a cumplir de las raíces fúlcreas, las cuales son más fuertes en los mangles menos jóvenes, y las mismas cumplen con la función de decantar la sal del agua de mar.

\section{Recomendaciones}

- $\quad$ El Plan de Manejo de Puerto El Morro, debe ser corregido en cuanto a la descripción botánica de Rhizophora mangle L., debiéndose indicar que la diferencia morfológica de las especies de mangle está dada por las características morfológicas propias de cada especie.

- $\quad$ Estudios de biología reproductiva de $R$. mangle deben ser realizados mínimo durante un año, abarcando los periodos con lluvia y otro sin lluvia.

- Las parcelas permanentes establecidas en el Estero Olmos y La Islita deben ser monitoreadas con el fin de proporcionar más datos de la biología reproductiva de $R$. mangle en los manglares El Morro.

- Observaciones fisonómicas deben realizarse periódicamente a fin de entender la dinámica poblacional de estos manglares y que esto sirva para los usuarios del sector para entender objetivamente si el manglar está o no en proceso de recuperación.

- $\quad$ Estudios de captura de carbono en los manglares de Puerto El Morro deberían ser realizados, para entender su función del servicio ambiental que prestan estos ecosistemas en cuanto a la calidad del aire.

\section{Bibliografía}

CABI. 2019. Compendio de especies invasoras. Francia. Obtenido de https://www.cabi.org/isc/datasheet/47509

Charcape-Ravelo, M. , y Moutarde, F. 2005. Diversidad florística y conservación del Santuario Regional de Piura Manglares San Pedro de VIce-Sechura. Peruana de Biología; Scielo Perú, 12(2). Obtenido de http://www.scielo.org.pe/scielo. php?script=sci_arttext\&pid=\$1727-99332005000200016

Cornejo, X. 2014 (ed.). Plants of the south American Pacific Mangrove Swamps (Colombia, Ecuador, Perú). Ed. Edu Quil, Universidad de Guayaquil, 312 p.

De la Cruz-Francisco, V. 2012. Rhizophora mangle Linnaeus, 1753 como especie sombrilla y razón biológica para la 
protección y restauración de la laguna de Tampamachoco, Veracruz, Mexico. BIOCYT Biología Ciencia y Tecnología, 5(18): 341-352.

Farnsworth, R., Ellison, A., y Gong, W. 1996. Elevated CO2 alters anatomy, physiology, growth, and reproduction of red mangrove (Rhizophora mangle L.). Springer - Oecologia, 599-609. doi:https://doi.org/10.1007/BF00329032

Kathiresan, K. y Bingham, L. B. 2001. Biología de manglares y ecosistemas de manglares. ELSEVIER, 40: 81-251.

Lozano Torres, Y. 2007. Los Sumideros de Carbono: Un análisis de la potencialidad económica en un bosque de manglar del Pacífico Colombiano. Ingeniería de Recursos Naturales y del Ambiente - Redalyc (6), 82-92. Obtenido de https:// www. redalyc.org/pdf/2311/231120826007.pdf

MAE. 2014. El Morro, un paraíso costero rodeado de mangles. Guayaquil. Obtenido de https://www.ambiente.gob.ec/elmorro-un-paraiso-costero-rodeado-de-mangles/

Ortiz-Reyes, A., Robles Lopez, K., Urrego Giraldo, L. E., y Romero Tabarez, M. (2018). Diversidad e interacciones biológicas en el ecosistema de manglar. Revista de Ciencias - Scielo, 22(2): 111-127.

Prance, G.T, 2018 Rhizophoraceae. Flora Neotropica. Vol.120. NYBG Press, Bronx, NY.

Raju, J. S. 2016. Reproductive biology of mangrove plants Clerodendrum inerme, Derris trifoliata, Suaeda maritima, Suaeda monoica, Suaeda nudiflora. The Wetlands Diversity", 31-68. doi:DOI: 10.1515/trser-2015-0092

Sánchez Nuñez, D. 2009. Patrones de floración, polinización y produccion de frutos de tres especies neotropicales de mangle presentes en humedales de san andres isla, caribe colombiano. Tesis de maestria, Universidad Nacional de Colombia, Departamento de Biologia, Bogotá. Obtenido de https: //core.ac.uk/download/pdf/11052844.pdf

Sofrony, C., Estrada-Castillo, S. M., y Martínez García, H. 2017. Plantas priorizadas para la conservación en la Región Caribe. Instituto de Investigación de Recursos Biológicos Alexander von Humboldt. doi:https://doi.org/10.15472/ rj6pz5

Soto, N. L. 2010. Un caso de tensiones sociales generados por las políticas ambientales: Refugio de Vida Silvestre "Manglares El Morro". Tesis de Maestría, Facultad Latinoamericana de Ciencias Sociales Sede Ecuador. Programa de estudios Socioambientales, Guayaquil. Obtenido de http://repositorio.flacsoandes.edu.ec:8080/ bitstream/10469/2485/4/TFLACSO-LMSN2010.pdf

Tarcila, L. N., y Machado, C. I. 2014. Polinización del viento y formación de propágulos en Rhizophora mangle L. (Rhizophoraceae): ¿limitación de recursos o polinización? Scielo, 86(1). doi:https://doi.org/10.1590/0001 37652014101712

Yánez-Arancibla, A. y Lara-Dominguez, A. 1999. Los manglares de América Latina en la encrucijada. Ecosistemas de Manglar en América Tropical, Instituto de Ecología AC. 9-16. 